\title{
Cross-reactivity of anti-programmed death ligand 2 polyclonal antibody in mouse tissues
}

\author{
ZHAO Yu ${ }^{1 \dagger}$, BIAN GanLan ${ }^{1 \dagger}$, YU CaiYong ${ }^{1}$, LIU FangFang ${ }^{1}$, LIU Ling ${ }^{1}$, GUO HongMin ${ }^{1}$, \\ GUO Jun ${ }^{2}$, JU Gong ${ }^{1 *} \&$ WANG Jian ${ }^{1 *}$ \\ ${ }^{1}$ Institute of Neurosciences, the Fourth Military Medical University, Xi'an 710032, China; \\ ${ }^{2}$ Department of Neurology, Tangdu Hospital, the Fourth Military Medical University, Xi'an 710038, China
}

Received June 25, 2012; accepted September 19, 2012; published online October 22, 2012

\begin{abstract}
The inhibitory co-receptor programmed death 1 (PD-1, encoded by $p d c d 1$ ) and its two ligands PD-L1 and PD-L2 comprise an important immune inhibitory signaling pathway for defense against microbes and for self-tolerance. Unlike other members of the B7-CD28 family, expression of PD-L1 and PD-L2 is not limited to the immune system. In this study, we determined that a polyclonal antibody (pAb) (R\&D Systems) against extracellular domains of mouse PD-L2 (mPD-L2) could recognize antigen(s) in diverse mouse tissues, including the anterior and intermediate pituitary gland, olfactory bulbs and olfactory epithelium, tongue epithelium, keratinized epithelial cells and skin and whisker hair follicles. These findings differed from previous reports of mPD-L2 localization. Reverse transcription PCR and Western blot analyses, however, were unable to detect any mPD-L2 transcripts or proteins of the $25-\mathrm{kD}$ predicted molecular weight in RNA and protein extracts, respectively, from the above tissues, suggesting that the anti-mPD-L2 pAb cross-reacts with certain novel antigen(s). Developmental studies revealed that the earliest expression of mPD-L2-like antigen was in the olfactory epithelium at embryonic day 12.5 (E12.5). At E14.5, mPD-L2-like antigen was present in the skin, tongue and follicles of the skin and whiskers. The distribution patterns of mPD-L2-like antigen remained similar from E18.5 to adulthood. The results of bioinformatic analysis and other experiments suggested neural cell adhesion molecule and hemicentin-1 as candidate proteins with cross-reactivity to the anti-mPD-L2 pAb. These results demonstrate that care is required in interpreting staining patterns generated when anti-PD-L2 pAb is used to locate PD-L2-expressing cells in the central nervous system and epithelial tissues, such as the olfactory epithelium. In addition, this anti-PD-L2 pAb may be used as an alternative antibody for labeling the olfactory epithelium during embryonic development in mice.
\end{abstract}

cross-reactivity, PD-L2, immunoreactivity, NCAM, hemicentin-1

Citation: Zhao Y, Bian G L, Yu C Y, et al. Cross-reactivity of anti-programmed death ligand 2 polyclonal antibody in mouse tissues. Sci China Life Sci, 2012, 55: 940-947, doi: 10.1007/s11427-012-4379-7

Signaling pathways involving the B7-CD28 family critically regulate the balance between the stimulatory and inhibitory signals needed for defense against microbes and for self-tolerance. These pathways provide secondary signals that can regulate the activation, inhibition, and fine-tuning of T-cell responses [1,2]. The programmed death-1 (PD-1)/

$†$ Contributed equally to this work

*Corresponding author (email: jwangfm@fmmu.edu.cn; jugong@fmmu.edu.cn)
PD-ligands pathway is one of the B7-CD28 pathways, comprising the PD-1 receptor and its two ligands, PD-L1 (also known as B7-H1 or CD274) and PD-L2 (also known as B7-DC or CD273). PD-1 belongs to the CD28 family and shares $23 \%$ amino acid sequence homology with cytotoxic T-lymphocyte-associated antigen 4 (CTLA-4) [2,3]. The extracellular region of PD-1 consists of a single IgV-like domain and its cytoplasmic region contains an immunoreceptor tyrosine-based inhibitory motif and an immunore- 
ceptor tyrosine-based switch motif. PD-1 is inducibly expressed on activated $\mathrm{T}$ cells, $\mathrm{B}$ cells and myeloid cells. PD-L1 and PD-L2 are type I transmembrane proteins with immunoglobulin $(\mathrm{Ig}) \mathrm{V}$ - and IgC-like domains in the extracellular region. PD-L1 is constitutively expressed in T cells, $\mathrm{B}$ cells, macrophages and dendritic cells. The expression of PD-L1 is also detected on non-lymphoid cells, such as endothelial cells in the heart, $\beta$ cells in the pancreas, glial cells in the inflamed brain and muscle cells [4-6], as well as in ovary, esophagus, kidney and brain tumor cells [7]. In contrast, PD-L2 expression has only been reported in activated macrophages and dendritic cells [1,7]. To the best of our knowledge, the possibility of PD-L2 expression in nervous tissues outside the immune system has not been investigated. In this study, we used a commercially available anti-mPD-L2 polyclonal antibody (pAb) (R\&D Systems, MN, USA) to detect the distribution of mPD-L2 expression in non-immune tissues, including adult mouse brain and embryonic tissues.

\section{Materials and methods}

\subsection{Ethics statement}

C57BL/6 mice (SLAC, Shanghai, China) were housed in a specific pathogen-free environment at the animal facility of the Institute of Neurosciences, the Fourth Military Medical University. All procedures were conducted according to the guidelines approved by the Fourth Military Medical University Animal Care and Use Committee (permit number: 082-2006). Mice were killed by decapitation or carbon dioxide asphyxiation, and all efforts were made to minimize suffering.

\subsection{Tissue preparation and staining}

(i) Fixation. Mice were anesthetized by intraperitoneal injection of an overdose of $1 \%$ sodium pentobarbital. The mice were then fixed by perfusion with $4 \%$ paraformaldehyde (PFA) in phosphate-buffered saline (PBS) via the aortic ventricle. The brain with intact olfactory bulbs and pituitary were carefully dissected. The dissected tissues were immersed in $20 \%$ sucrose-PBS at $4^{\circ} \mathrm{C}$ until they sank to the bottom of the container.

(ii) Decalcification. Adult mouse olfactory epithelium was prepared as described previously [8], with modifications. Briefly, after fixation, the head of the mouse was submersed in $10 \%$ EDTA until the bone became half-transparent and soft when tested by application of needle tracks. The decalcified brain was then placed in a $20 \%$ sucrose solution until the brain tissue was dense enough to sink to the bottom of the container.

(iii) Immunohistochemistry (IHC). Tissue sections were cut at $10 \mu \mathrm{m}$ using a cryostat and mounted on poly-Dlysine-coated glass slides. The sections were incubated with the primary antibody diluted in $1 \%$ bovine serum albumin solution at $4^{\circ} \mathrm{C}$ overnight. After washing three times with PBS, the sections were incubated with fluorescein-conjugated secondary antibody for $2 \mathrm{~h}$ at room temperature. The sections were observed and photographed under a BX51 microscope (Olympus, Japan). Mouse embryos at embryonic days 10.5 (E10.5), E12.5, E14.5, E16.5, E18.5 and post-natal day (P) 1 were fixed with $4 \%$ PFA in PBS at $4^{\circ} \mathrm{C}$ overnight, soaked in $25 \%$ sucrose-PBS at $4^{\circ} \mathrm{C}$ overnight and embedded in Optimal Cutting Temperature compound. Frozen sections (10 $\mu \mathrm{m}$ thickness) were prepared as described above.

(iv) Whole-mount IHC (WMIHC) of embryos. E10.5E12.5 embryos were fixed with freshly prepared methanol/dimethylsulfoxide $/ \mathrm{H}_{2} \mathrm{O}_{2}$ at $4{ }^{\circ} \mathrm{C}$ for $5-10 \mathrm{~h}$. Embryos were blocked with blocking reagents (PBSMT, 2\% skim milk, $0.5 \%$ Triton X-100 in PBS) for $2 \mathrm{~h}$ with rocking and incubated with goat anti-PD-L2 pAb diluted in PBSMT at $4{ }^{\circ} \mathrm{C}$ overnight. Embryos were washed five times with rocking in PBSMT for at least $1 \mathrm{~h}$ between washes, and then incubated with the biotinylated donkey anti-goat $\operatorname{IgG}$ antibody in PBSMT at $4^{\circ} \mathrm{C}$ overnight. The embryos were washed as before and incubated with avidin-biotin complexes diluted in PBSMT at $4^{\circ} \mathrm{C}$ overnight. After washing, the embryos were incubated in diaminobenzidine $/ \mathrm{NiCl}_{2}$ at room temperature for $30 \mathrm{~min} . \mathrm{H}_{2} \mathrm{O}_{2}$ was added to a final concentration of $0.03 \%$ and rocked until the color intensity in the embryos appeared well-developed before background staining emerged. The results were photographed under a STZ10 stereomicroscope (Olympus).

\subsection{Antibodies}

Two lots of goat pAbs against mPD-L2 were purchased from R\&D Systems (MN, USA). The catalog and lot numbers were AF1022, GVZ012091 and AF1022, GVZ016041, respectively. Rat monoclonal antibody (mAb) against mPD-L2 (clone: TY25) was purchased from eBioscience (CA, USA). Goat pAb against mPD-L1 was also purchased from R\&D Systems (catalog number AF1019, lot number GGF016021). Rat anti-NCAM mAb was purchased from Abcam (UK). Fluorescein isothiocyanate (FITC)- and TexRed-conjugated donkey antibodies against goat IgG and FITC-conjugated goat antibody against rat IgG were purchased from Jackson ImmunoResearch (PA, USA). Biotinylated rabbit anti-rat IgG or anti-goat IgG and streptavidin horseradish peroxidase (HRP) were purchased from Sigma-Aldrich (MO, USA).

\subsection{BLAST search and mRNA analysis by RT-PCR}

BLAST searches were performed within the National Center for Biotechnology Information (NCBI) non-redundant database using the amino acid sequences of the mPD-L2 extracellular domains, excluding the signal-peptide frag- 
ments. Candidate molecules, including butyrophilin-like 2 (BTNL2), Skint1, hemicentin-1 and NCAM, which showed some degrees of identity with mPD-L2 by BLAST analysis, were subsequently chosen for detection in various tissues by PCR.

Total RNA was prepared from organs including the olfactory bulb, olfactory membrane, pituitary, skin, tongue and brain, using the Rneasy Micro Kit (TaKaRa, Japan). Fixed amounts of RNA were reverse transcribed into cDNA with reverse transcriptase (TaKaRa). The following primers were used for PCR detection of PD-L2, BTNL2, Skint1 and hemicentin-1: PD-L2 forward, 5'-CTGAACTGGAAGGGATAAGAGC-3' and reverse, 5'-GAAGATCAAAGCGATGGTGC-3'; BTNL2 forward, 5'-ATGGAGGACAGCACTGAAGAGGG-3' and reverse, 5'-TCCGAAGTTCTGGCATTGTGAAT-3'; Skint1 forward, 5'-TCCACCACAACAAGCCCAGCAC-3' and reverse, 5'-CGTGGGAACCAACCTCCAGAGT-3'; hemicentin-1 forward, 5'-ATGATTGCCCAGGAAGTGGTCC-3' and reverse, 5'-TGGATAAGCTGCAGCACCTCAT- $3^{\prime}$. The PCR conditions consisted of 35 cycles at $95^{\circ} \mathrm{C}$ for $30 \mathrm{~s}, 58^{\circ} \mathrm{C}$ for $30 \mathrm{~s}$, and $72^{\circ} \mathrm{C}$ for 1 min. PCR products were analyzed by agarose gel electrophoresis.

\subsection{Western blotting}

Crude olfactory bulb, head (without brain tissue), skin and brain (without olfactory bulbs) extracts were prepared using a Polytron homogenizer (FLUKO, Germany) in lysis buffer comprising $150 \mathrm{mmol} \mathrm{L}^{-1} \mathrm{NaCl}, 20 \mathrm{mmol} \mathrm{L}^{-1}$ Tris- $\mathrm{HCl}(\mathrm{pH}$ 7.4), $5 \mathrm{mmol} \mathrm{L}{ }^{-1}$ EDTA, $1 \% \mathrm{NP} 40$, and protease inhibitor cocktail (Complete ${ }^{\mathrm{TM}}$, Roche, UK), separated by SDSPAGE, and transferred onto Hybond-P filters (Amersham Biosciences Co., NJ, USA). Filters were incubated with anti-mPD-L2 $(1: 100)$ and anti-NCAM $(1: 200)$ antibodies at $4{ }^{\circ} \mathrm{C}$ overnight and visualized using an HRP-labeled anti-mouse IgG Ab (CWbio, China) with the enhanced chemiluminescence system (Amersham Biosciences Co., NJ, USA).

\section{Results}

\section{1 mPD-L2 pAb reacts with antigens in the olfactory} epithelium, olfactory bulbs and pituitary gland

Immunofluorescent staining of brain sections with antimPD-L2 pAb (catalog number AF1022, lot number GVZ012091) yielded unexpected results. The glomerular layers of the olfactory bulbs (Figure $1 \mathrm{~A}$ and $\mathrm{C}$ ) and accessory olfactory bulbs (Figure $1 \mathrm{~B}$ and $\mathrm{C}$ ) were mPD-L2positive. Meanwhile, the anti-mPD-L2 pAb stained the anterior (AP) and intermediate (IP) lobes, but not the posterior lobe, of the pituitary (Figure 1D). Most cells in the AP and IP gland were mPD-L2-positive. The ventral surface of the medium eminence was strongly mPD-L2-positive, extend-

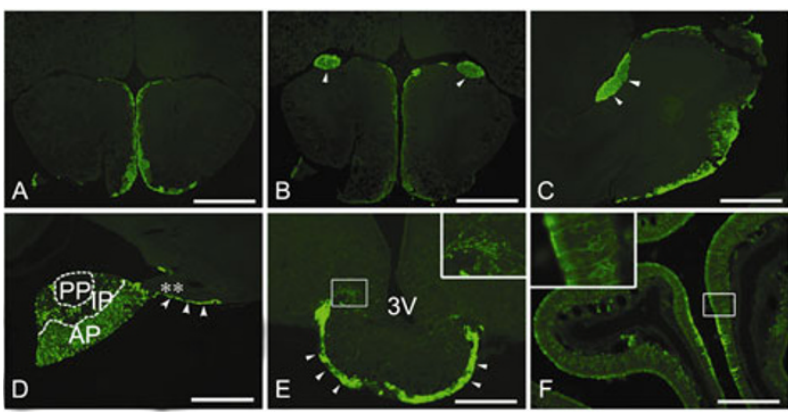

Figure 1 Immunostaining patterns of anti-mPD-L2 $\mathrm{pAb}$ in the adult olfactory system and pituitary gland. A, mPD-L2-positive signals in the glomerular layer of olfactory bulbs. B, mPD-L2-positive signals in accessory olfactory bulbs (arrow heads) in the front sections of the brain. C, mPD-L2-positive signals in the glomerular layer of olfactory bulbs and accessory olfactory bulbs (arrow head) in a sagittal section. D, $\mathrm{mPD}-\mathrm{L} 2$-positive signal distributions in anterior and intermediate pituitary (AP and IP) and the most ventral part of the pituitary stem (**) in a sagittal section (arrowheads). E, mPD-L2-positive signals in median eminence (EM). Strong fluorescence surrounded the EM (arrowheads) and densely positive nerve fibers were also seen in the bottom of the third ventricle (3V). The boxed area shows the enlarged mPD-L2-positive nerve fibers at the up-right corner of panel E. F, mPD-L2-positive signals in the olfactory epithelium. The boxed area shows the enlarged typical bipolar olfactory neurons at the upper-left corner of panel F. Scale bar, $50 \mu \mathrm{m}$ in A, B, C, D and $100 \mu \mathrm{m}$ in $\mathrm{E}, \mathrm{F}$.

ing to the ventral medial hypothalamus. A group of mPDL2-positive varicose terminal nerve fibers could be identified medial to the hypothalamic extension, which seemed to be converging towards the hypothalamic extension (Figure 1E). mPD-L2-positive signals also extended onto the ventral surface of the pituitary stalk (Figure 1D). The presence of mPD-L2 in the accessory olfactory bulb and glomerular layer of the olfactory bulb suggested that the olfactory epithelium, in which the olfactory primary sensory neurons are located, might also be mPD-L2-positive. Because the convoluted olfactory epithelium is located at the roof of the posterior nasal cavity, decalcified head tissue was used. mPD-L2-positive olfactory epithelium lining the nasal cavity and typical bipolar olfactory sensory neurons in the olfactory epithelium could be identified (Figure 1F). However, we simultaneously stained the above-mentioned tissues with anti-mPD-L1 pAb and were unable to detect any mPD-L1 signals (data not shown). These results demonstrated that mPD-L2-positive signals were distributed in the olfactory epithelium, olfactory bulbs and pituitary gland.

\subsection{Developmental characterization of mPD-L2 pAb cross-reactivity in mice}

We mapped the distribution of mPD-L2 in mice at different developmental stages, to help characterize the antigens that reacted with the anti-mPD-L2 pAb. Embryos at E10.5, E12.5, E14.5, E18.5 and neonatal mouse tissues at P1 were prepared, sectioned and incubated with anti-mPD-L2 pAb. 
E10.5 and E12.5 embryos were also processed for WMIHC in situ.

No detectable signals were found in WMIHC or IHC sections at E10.5 (Figure 2A). At E12.5, however, mPD-L2positive olfactory nerve bundles from the nasal cavity projecting to the olfactory bulbs were clearly identified in both WMIHC and IHC sections (Figure 2B-D). These nerve bundles targeted the outer layer of the olfactory bulbs, appearing as a curved shape in sagittal sections (Figure 2D). The epidermis of the mouth and nostrils was also mPD-L2positive at this stage (Figure $2 \mathrm{~B}$ and $\mathrm{C}$ ). mPD-L2-positive signals along the oral cavity and tongue surface in WMIHC were confirmed by immunofluorescence staining in E12.5 embryonic sections (Figure 2D). mPD-L2-positive signals began to appear in the olfactory epithelium, olfactory nerve bundles and olfactory bulbs at E12.5, and the epithelia of the oral cavity and tongue also displayed light mPD-L2positive signals.

mPD-L2-positive signals further increased at E14.5, and strong immunofluorescence signals were observed on the surfaces of the head, nasal and oral cavities (Figure 2E). The typical olfactory bipolar neurons in the olfactory epithelium were strongly mPD-L2-immunoreactive. Bipolar cells extending dendrites to the epithelial surface from the
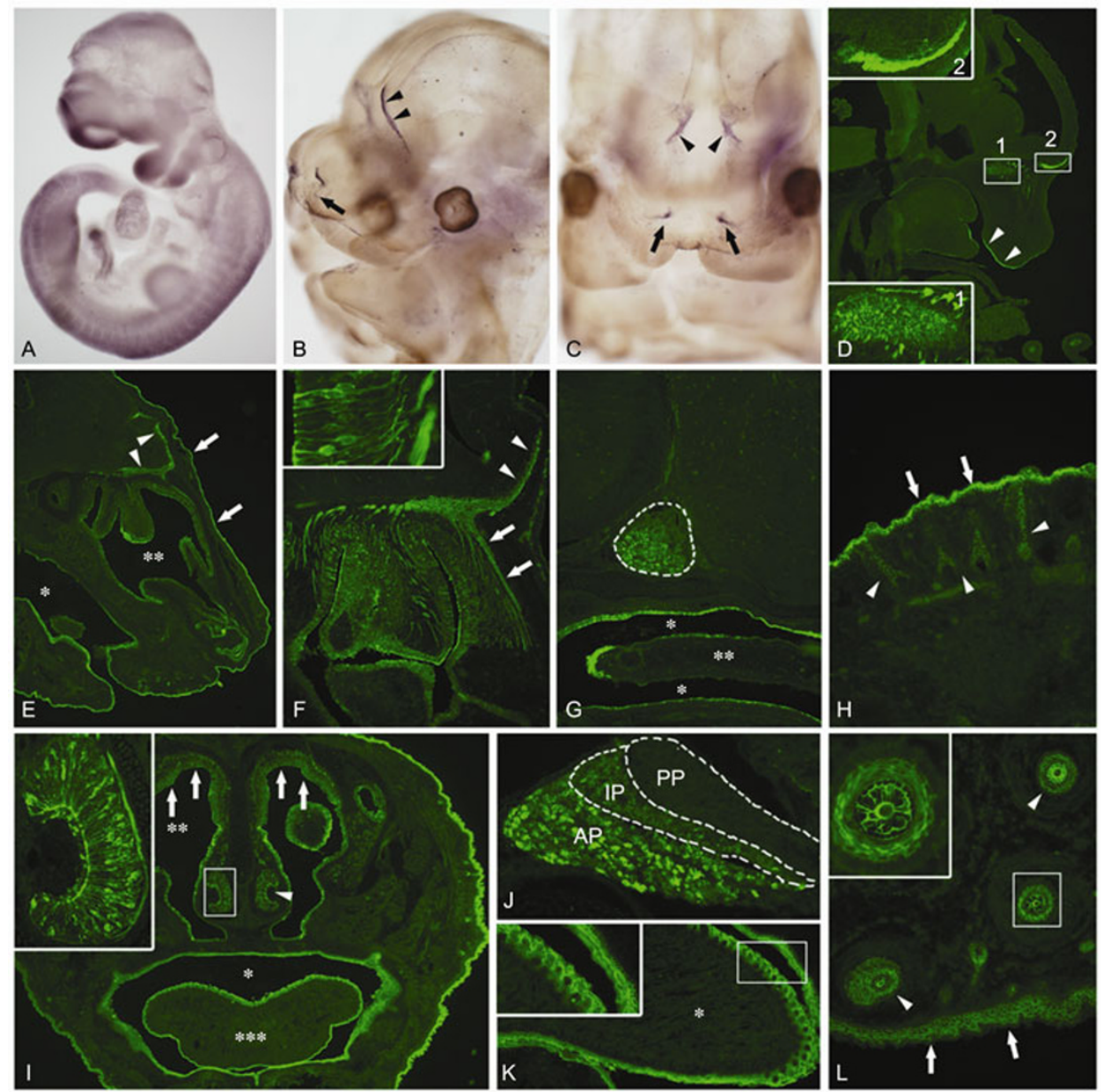

Figure 2 Immunostaining patterns of mPD-L2 immunoreactivity at different developmental stages. A, No mPD-L2-positive signals were seen with WMIHC at E10.5. B and C, Side and front views of the embryonic head with WMIHC at E12.5. mPD-L2-positive signals were found in the olfactory bulbs (arrow head) and skin (arrow). D, Immunostaining in a sagittal section of E12.5 shows that the olfactory bulb and olfactory epithelium were mPD-L2-positive (box 1 and 2, respectively). The skin at the oral edge displayed mPD-L2-positive signals (arrow head). Boxed areas 1 and 2 are enlarged at the upper and bottom corners of the panel, respectively. E, Immunostaining in a head sagittal section of E14.5. The olfactory bulbs (arrow head), olfactory epithelium lining the top of the nasal cavity $(* *)$, epithelium lining the oral cavity $(*)$ and skin (arrow) were mPD-L2-positive. F, Olfactory epithelium at E14.5 was mPD-L2-positive. Olfactory bulb (arrow head) and olfactory nerve bundles (arrow) showed strong immunostaining. The top boxed area shows an olfactory neuron with a typical bipolar shape. G, The primordium of the pituitary gland (dash-line circle), epithelia of oral cavity $(*)$ and tongue $(* *)$ were mPD-L2-positive at E14.5. H, The epidermis (arrow) and hair follicles (arrow head) were mPD-L2-positive at E14.5. I, mPD-L2 immunostaining pattern of a frontal head section at E18.5. The epidermis, epithelia lining the oral $(*)$ and nasal $(* *)$ cavities, epithelium of tongue $(* * *)$ and vomeronasal sensory system (arrow head and boxed) were mPD-L2-positive. The boxed vomeronasal epithelium is enlarged at the corner of the panel. J, Immunostaining pattern of mPD-L2-positive signals in the pituitary at E18.5. Dashed lines separate the posterior pituitary (PP), intermediate pituitary (IP) and anterior pituitary (AP). mPD-L2-positive signals were present in the AP and IP but not in PP. K, The tongue epithelium was mPD-L2-positive at E18.5. The boxed area is enlarged at the corner of the panel. L, Whisker follicles (arrow heads) and epidermis (arrows) were mPD-L2-positive at E18.5. The boxed area is enlarged at the corner of the panel. 
apical pole were characterized by the presence of dendritic knobs and numerous cilia (Figure 2F). The olfactory sensory neuron axons fasciculated upon exiting the basal side of the olfactory epithelium and traversed the cribiform plate to form the outer layer of the olfactory bulbs (Figure 2F). In addition to the olfactory system, the primordial pituitary was beginning to display mPD-L2-positive staining at E14.5 (Figure 3G). The oral and tongue epithelia displayed obvious mPD-L2-positive signals at E14.5 compared to their weak signals at E12.5. At this developmental stage, skin and hair follicles showed obvious mPD-L2-immunoreactivity (Figure 2H).

At E18.5, the same staining pattern observed in the olfactory sensory neurons was also clearly present in the vomeronasal sensory neurons in coronal sections (Figure 2I). The mPD-L2-positive signal pattern was similar to that in the adult pituitary, in which the posterior pituitary (PP) was negative while the AP and IP contained numerous mPD-L2-positive cells (Figure 2J). Various epithelia in the head, including the skin, and epithelia of the tongue and oral cavity, displayed strong fluorescence signals at E18.5 (Figure $2 \mathrm{~K}$ and $\mathrm{L}$ ). Although epithelial cells surrounding the taste buds were positive, no detectable signals were found on the surfaces of the sensory cells in the taste buds. Fluorescence signals were observed in the keratinized epithelial cells of the skin. In addition to its distribution in the skin, specific fluorescence was also detected in the hair follicles of the skin and whiskers (Figure 2L). Overall, these results demonstrated that mPD-L2-positive signals first appeared around E12.5 in the olfactory epithelium and olfactory bulbs, and then gradually extended to other epithelia in subsequent developmental stages. The distributions of mPD-L2 immunostaining patterns in tissues were similar from E14.5 to P1 until adulthood (data not shown).

\subsection{Cross-reactivity of anti-mPD-L2 pAb with anti- gen(s) other than mPD-L2}

Some authors found no mPD-L2 mRNA in brain tissue by Northern blotting [8]. Given that the mPD-L2 molecule was reported to be distributed mainly in organs of the immune system, we suspected that the mPD-L2-positive staining in mouse brain and diverse epithelia might be due to cross-reactivity with proteins other than mPD-L2. To exclude the possibility of differences in antibody quality between lots, we repeated the immunostaining experiments with a second batch of the pAb (R\&D Systems; catalog number AF1022; lot number GVZ016041), and acquired similar results (data not shown). To further test our assumption, we also purchased a mAb against mPD-L2 from eBioscience, which has been used for IHC in other studies [5]. No positive signals in the adult brain or developing embryos were detected with this mAb (data not shown). RT-PCR also failed to detect any mPD-L2 mRNA transcripts in the brain, head, skin and tongue extracts of mice (data not shown). These results therefore confirmed that the anti-mPD-L2 pAb from R\&D Systems cross-reacted with antigens other than mPD-L2 itself.

To identify the proteins that could be targeted by the anti-PD-L2 pAb, a BLAST database search was performed using the 20-241 amino acid sequence fragment of mPD-L2 containing the extracellular domains, excluding its signal sequence. Several homologs of mPD-L2 with different sequence identities (Id) were found. Among these homologs, NCAM (Id about 30\%), hemicentin-1 (Id about 27\%), Skint1 (Id about 28\%) and BTNL2 (Id about 28\%) were chosen for further confirmation, based on their structures and identities.

Western blotting showed that anti-NCAM could recognize 180-, 140- and 120-kD bands in olfactory bulbs and brain tissue extracts, while only a $140-\mathrm{kD}$ band was present in the skin and head (without brain tissue) extracts (Figure $3 \mathrm{~A}$, left panel). The anti-PD-L2 pAb could also recognize proteins of around $180 \mathrm{kD}$, but not the $140-$ and $120-\mathrm{kD}$ bands. In addition, the anti-PD-L2 pAb recognized several bands much larger than $180 \mathrm{kD}$ (Figure 3A, right panel). However, no band appeared around $25 \mathrm{kD}$, which is the predicted molecular weight (MW) of mPD-L2 (data not shown). These results suggested that the anti-mPD-L2 pAb could bind several proteins with different MWs.
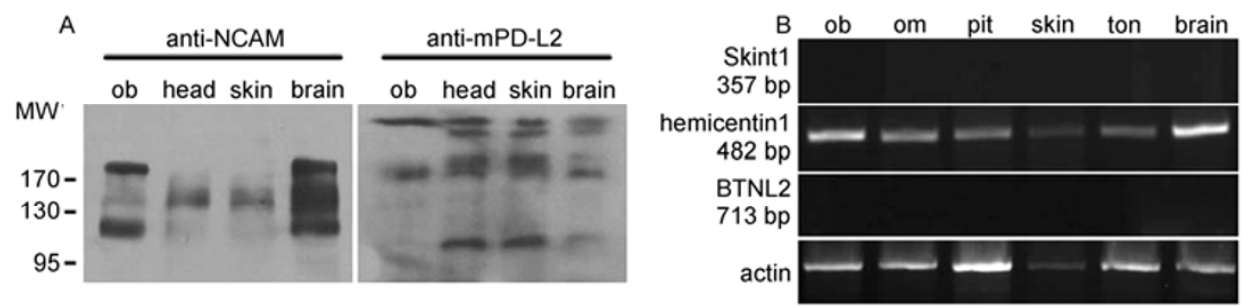

Figure 3 Western blot and RT-PCR analyses. A, Western blot analysis of extracts from the olfactory bulbs (ob), head (without brain tissue), skin and brain (without olfactory bulbs) with anti-NCAM mAb (left panel) and anti-mPD-L2 pAb (right panel). Left panel: 180-, 140- and 120-kD bands were recognized by the NCAM mAb in the brain and olfactory bulb extracts. However, the head and skin lanes only showed a definitive band around $140 \mathrm{kD}$ and a weak $120-\mathrm{kD}$ band. Right panel: Using anti-mPD-L2 pAb, bands in addition to that around $180 \mathrm{kD}$ appeared in all tissue lanes, with several bands much larger than $180 \mathrm{kD}$. B, RT-PCR detected Skint1, hemicentin-1 and BTNL2 transcripts from the olfactory bulb (ob), olfactory membrane (om), pituitary (pit), skin, tongue (ton) and brain tissue RNA extracts. 
RT-PCR detected hemicentin-1 mRNA transcripts, but not Skint1 and BTNL2 transcripts, in the olfactory bulbs, olfactory epithelium, skin, tongue and brain tissue extracts (Figure 3B). These results suggested that NCAM and hemicentin-1 may be the novel antigens recognized by the anti-mPD-L2 pAb.

\subsection{Anti-mPD-L2 pAb and anti-NCAM mAb immu- noreactivities co-localize within the olfactory system}

NCAM is one of the best-characterized members of the Ig superfamily. It is widely expressed in olfactory sensory neuron bodies and processes and plays important roles in the olfactory system. IHC analysis showed a high degree of overlap between the immunoreactivities of the anti-NCAM $\mathrm{mAb}$ and mPD-L2 $\mathrm{pAb}$ in the olfactory bulbs (Figure 4A, $\mathrm{A}^{\prime}, \mathrm{A}^{\prime \prime}$ ), accessory olfactory bulbs (Figure $4 \mathrm{~B}, \mathrm{~B}^{\prime}, \mathrm{B}^{\prime \prime}$ ), olfactory sensory neurons (Figure $4 \mathrm{C}, \mathrm{C}^{\prime}, \mathrm{C}^{\prime \prime}$ ) and the vomer-

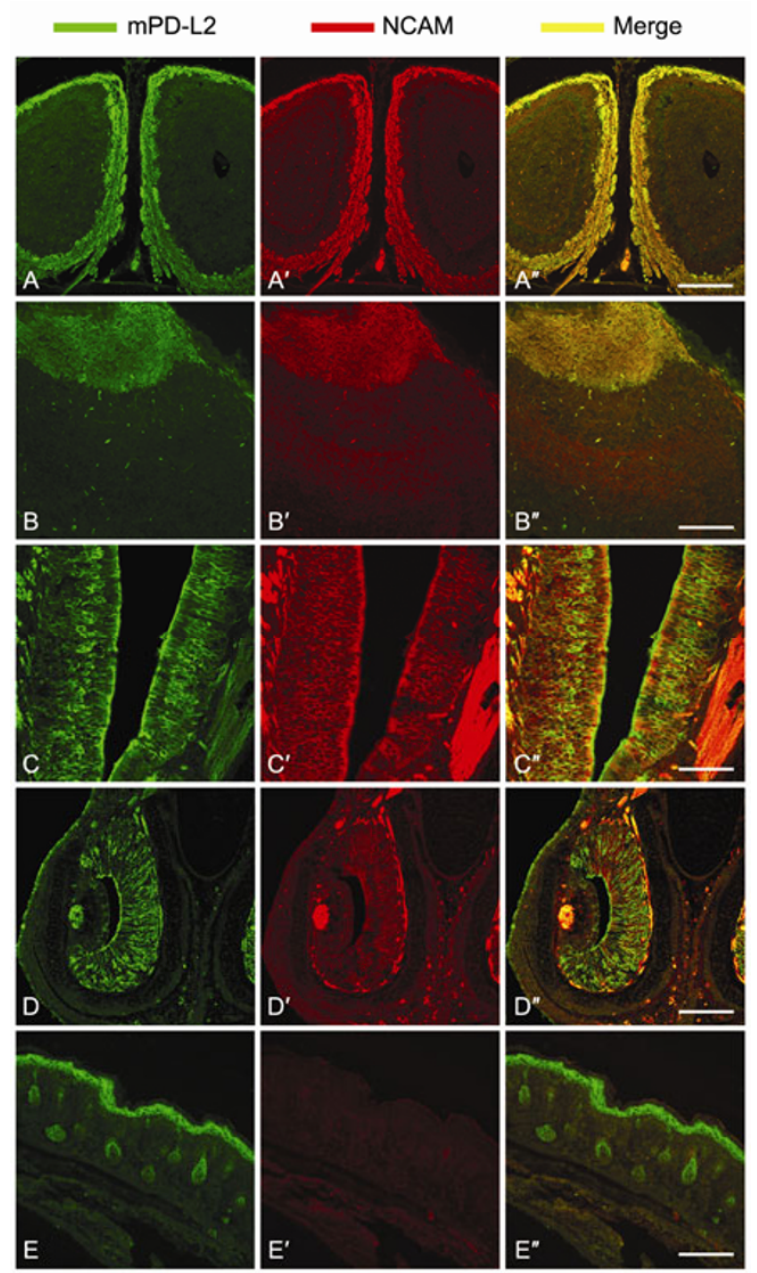

Figure 4 Double-staining with anti-PD-L2 pAb (green) and anti-NCAM $\mathrm{mAb}$ (red) in sections of adult mouse tissues. A, Olfactory bulb. B, Accessory olfactory bulbs. C, Olfactory epithelium. D, Vomeronasal sensory system. E, Epidermis and hair follicles. In the olfactory system, mPD-L2 and NCAM signals were highly co-localized (yellow) except in epidermis and hair follicles. Scale bar, $100 \mu \mathrm{m}$. onasal sensory neurons (Figure 4D, D', D"). However, although skin and hair follicles were positive for anti-mPD-L2 $\mathrm{pAb}$ staining, they were negative for anti-NCAM mAb staining (Figure 4E, E', E").

\section{Discussion}

The two PD-1 ligands differ in their expression patterns, with PD-L2 expression being much more restricted than that of PD-L1 [9]. A variety of normal nonlymphoid tissues express PD-L2 transcripts, with high levels of expression in liver, low expression levels in the lung and kidney, and no expression in the brain $[9,10]$. However, transcript levels do not always correlate with protein levels $[6,11]$. In particular, PD-L2 protein is rarely detected in nonlymphoid organs under normal conditions because of post-transcriptional regulation [7]. PD-L2 is inducibly expressed in dendritic cells, macrophages and cultured bone marrow-derived mast cells [10]. In this study, the mPD-L2-positive signals distributed in nonlymphoid organs were thus highly suspected to result from cross-reaction with other antigens, based not only on previous studies, but also on the current results with mPD-L2 mAb in Western blot and RT-PCR experiments.

Four proteins, BTNL2, Skint1, NCAM and hemicentin-1, were originally identified from the NCBI database based on a bioinformatic search for homologous proteins. These proteins are all members of the Ig superfamily. BTNL2 is a butyrophilin family member with homology to B7 costimulatory molecules [12]. Analysis of BTNL2 in normal tissue showed that it is expressed in thymus, spleen, lymph node, stomach, small intestine, cecum, lung and large intestine [12]. Skintl is a newly-identified gene expressed in thymus and skin that encodes a protein with an Ig-like domain, and which shows the greatest similarity to the butyrophilin gene. It encodes a protein of 364 amino acids with a predicted MW of $46 \mathrm{kD}$. RT-PCR of RNA from various tissues revealed robust expression of Skintl only in thymus and skin, and specifically in thymic epithelial cells and keratinocytes. Skint1 is expressed at E15 and continues into adulthood [13]. In this study, however, the characteristic expression patterns of BTNL2 and Skint1 differed from the mPD-L2 pAb immunoreactivity, and RT-PCR analysis also failed to detect BTNL2 and Skint1 transcripts in diverse epithelia and nervous tissues. These results suggest that BTNL2 and Skint1 do not cross-react with mPD-L2.

Hemicentins are a family of secreted extracellular matrix components first identified in Caenorhabditis elegans, with two orthologs in most vertebrate genomes, including humans and mice [14]. Hemicentins are characterized by a single, highly conserved von Willebrand A domain at the amino terminus, a long stretch $(>40)$ of tandem Ig domains, multiple tandem epidermal growth factor domains, and a single fibulin-like carboxyl-terminal module [14,15]. Hemicentin in C. elegans is composed of 5198 amino acids (>600 
$\mathrm{kD}$ ), and organizes hemidesmosome-mediated mechanosensory neuron and uterine attachments to the epidermis. Hemicentin forms an oriented track-like geometry with the apparent function of constricting broad regions of cell contact into discrete, linear junctions [14].

In mouse developmental stages from E12.5, hemicentin- 1 expression can be observed in a pericellular pattern around individual epithelial cell surfaces. At E14.5, anti-hemicentin-1 antibodies stain the periphery of basal and suprabasal keratinocytes and tongue epithelium, and by E17.5, hemicentin-1 staining is detected in hair and whisker follicles [16]. The characteristic expression pattern of hemicentin-1 during mouse development is very similar to that of mPD-L2. It therefore seems likely that hemicentin-1 may cross-react with the anti-mPD-L2 pAb in mouse tissues.

The majority of cell adhesion molecules that have been identified in the nervous system fall into one of three families based on their sequence structures: the Ig superfamily, the integrins, and the cadherins [17]. The Ig superfamily can be subdivided into at least five groups of related molecules based on the number of Ig-like domains, fibronectin type III-like repeats and the mode of membrane attachment: NCAM, L1, TAG-1, myelin-associated glycoprotein and P0 [17]. Three major isoforms of NCAM are generated via alternative splicing of a primary transcript from a single gene. Two isoforms are transmembrane forms of 140 and $180 \mathrm{kD}$, respectively, and the third $(120 \mathrm{kD})$ is attached to the cell membrane via a glycophosphatidyl inositol linkage [18]. The amino acid sequences of NCAMs are highly conserved among vertebrate species.

During the embryonic and early postnatal development of the olfactory system in the mouse, NCAM was detectable in the developing olfactory epithelium, but not in regions developing into the respiratory epithelium [19]. Among the three isoforms of NCAM, NCAM-180 (isoform of $180 \mathrm{kD}$ ) is strongly expressed on neurons in the developing olfactory system. At E10.5, the upper medial portions of the nasal pit and axons leaving (from) the neural epithelium were NCAM-180-positive, and at E13.5, NCAM-180 was expressed in the cell bodies of sensory neurons and their dendrites. Moreover, axons of the sensory neurons are strongly NCAM-180 immunoreactive in the nerve-fiber layer and in the glomeruli of the olfactory bulb [19]. This NCAM-180 expression pattern in the olfactory system was similar to the anti-mPD-L2 pAb immunostaining pattern in the current study. The recognition of a band around $180 \mathrm{kD}$ in olfactory bulb extracts by anti-mPD-L2 pAb in Western blots and the co-localization of mPD-L2-positive signals and NCAM immuoreactivities in olfactory bulbs and epithelia suggests that that anti-mPD-L2 pAb might cross-react with NCAM180 in the olfactory system.

Several reports have demonstrated the cross-reactivity of both mAbs and pAbs in IHC studies. mAb CD133-2 clone AC141 against hematopoietic stem cell antigen CD133 showed cross-reactivity with cytokeratin 18 [20] in cryostat sections, but not in paraffin sections. The use of AC141 to identify CD133-expressing cells in cryostat sections could thus be misleading [20]. A commercial goat anti-mouse GATA-4 pAb (GMP) used to label Sertoli nuclei gave unexpected results by labeling the complete development of the acrosome, from its assembly from fused Golgi vesicles to the lateral expansion of the acropaxome, which covers the spermatid nucleus [21]. These results were confirmed using a different batch of the commercial antibody. This fortuitous labeling of acrosome development with GMP antibody provides an alternative to the use of peanut and soybean agglutinin lectin histochemistry to label acrosomes [21]. This reflects the current study, where, although pAbs are well-known to cross-react with several epitopes and are prone to batch-to-batch variability, labeling of the developing olfactory epithelium with anti-PD-L2 pAb from R\&D Systems was replicated with a different batch from the supplier.

In conclusion, our study showed that the anti-mPD-L2 $\mathrm{pAb}$ tested here cross-reacts with several antigens in developing and adult mouse tissues. Care is therefore needed in interpreting the staining patterns obtained when the antiPD-L2 pAb (R\&D Systems) is used to identify PD-L2expressing cells in the central nervous system and epithelial tissues, such as the olfactory epithelium. However, these results also indicate that this anti-PD-L2 pAb can be used as an alternative antibody for labeling the olfactory epithelium during embryonic development in mice.

We thank Ms. Qiu JianYong and Fei LingLing for their technical assistance. This work was partially supported by the Scientific Research Foundation for the Returned Overseas Chinese Scholars, Ministry of Education of China (Grant No. HG3102) and National Natural Science Foundation of China (Grant No. 81100899).

1 Keir M E, Francisco M L, Sharpe A H. PD-1 and its ligands in T-cell immunity. Curr Opin Immunol, 2007, 19: 309-314

2 Okazaki T, Honjo T. The PD-1-PD-L pathway in immunological tolerance. Trends Immunol, 2006, 27: 195-201

3 Ishida Y, Agata Y, Shibahara K, et al. Induced expression of PD-1, a novel member of the immunoglobulin gene superfamily, upon programmed cell death. EMBO J, 1992, 11: 3887-3895

4 Dong H, Zhu G, Tamada K, et al. B7-H1, a third member of the B7 family, co-stimulates T-cell proliferation and interleukin-10 secretion. Nat Med, 1995, 5: 1365-1369

5 Salama A D, Chitnis T, Imitola J, et al. Critical role of the programmed death-1 (PD-1) pathway in regulation of experimental autoimmune encephalomyelitis. J Exp Med, 2003, 198: 71-78

6 Ishida M, Iwai Y, Tanaka Y, et al. Differential expression of PD-L1 and PD-L2, ligands for an inhibitory receptor PD-1, in the cells of lymphohematopoietic tissues. Immunol Lett, 2002, 84: 57-62

7 Okazaki T, Honjo T. The PD-1 and PD-1 ligands: from discovery to clinical application. Int Immunol, 2007, 19: 813-824

8 Robinson D A, Bogdanffy M S, Reed C J. Histochemical localisation of carboxylesterase activity in rat and mouse oral cavity mucosa. Toxicology, 2002, 180: 209-220

9 Latchman Y, Wood C R, Chernova T, et al. PD-L2 is a second ligand for PD-1 and inhibits T cell activation. Nat Immunol, 2001, 2: 261268 
10 Sharpe A H, Wherry E J, Ahmed R, et al. The function of programmed cell death 1 and its ligands in regulation autoimmunity and infection. Nat Immunol, 2007, 8: 239-245

11 Liang S C, Latchman Y E, Buhlmann J E, et al. Regulation of PD-1, PD-L1 and PD-L2 expression during normal and autoimmune responses. Eur J Immunol, 2003, 33: 2706-2716

12 Arnett H A, Escobar S S, Gonzalez-Suarez E, et al. BTNL2, a butyrophilin/B7-like molecule, is a negative costimulatory molecule modulated in intestinal inflammation. J Immunol, 2007, 178: 15231533

13 Boyden L M, Lewis J M, Barbee S D, et al. Skint1, the prototype of a newly identified immunoglobulin superfamily gene cluster, positively selects epidermal $\gamma \delta$ T cell. Nat Genet, 2008, 40: 656-662

14 Vogel B E, Hedgecock E M. Hemicentin, a conserved extracellular member of the immunoglobulin superfamily, organizes epithelial and other cell attachments into oriented line-shaped junctions. Development, 2001, 128: 883-894

15 Dong C, Muriel J M, Ramirez S, et al. Hemicentin assembly in the extracellular matrix is mediated by distinct structural modules. J Bio
Chem, 2006, 281: 23606-23610

16 Xu X, Dong C, Vogel B E. Hemicentins assemble on diverse epithelia in the mouse. J Histochem Cytochem, 2007, 55: 119-126

17 Zhang Y, Yeh J, Richardson P M, et al. Cell adhesion molecules of the immunoglobulin superfamily in axonal regeneration and neural repair. Restorat Neurol Neurosci, 2008, 26: 81-96

18 Crossin K L, Krushel L A. Cellular signaling by neural cell adhesion molecules of the immunoglobulin superfamily. Dev Dyn, 2000, 218 : 260-279

19 Miragall F, Kadmon G, Schachner M. Expression of L1 and N-CAM cell adhesion molecules during development of the mouse olfactory system. Dev Biol, 1989, 135: 272-286

20 Pötgens A J, Schmitz U, Kaufmann P, et al. Monoclonal antibody CD133-2 (AC 141) against hematopoietic stem cell antigen CD133 shows crossreactivity with cytokeratin 18. J Histochem Cytochem, 2002, 50: 1131-1134

21 McClusky L M, Patrick S, Barnhoorn I E, et al. Immunohistochemical study of nuclear changes associated with male germ cell death and spermiogenesis. J Mol Histol, 2009, 40: 287-299

Open Access This article is distributed under the terms of the Creative Commons Attribution License which permits any use, distribution, and reproduction in any medium, provided the original author(s) and source are credited. 